Corresponding author: Yolanda Segovia Huertas, Department of Biotechnology, Alicante University, Alicante, Spain

Email: Yolanda.segovia@ua.es

Telephone number: +34-96-590-3852/ +34-96-590-3999; Fax numbers: +34-96-590-3965

\title{
Ultrastructural study of retinal development in the turtle Trachemys scripta elegans
}

Segovia, Y.; García, M.; Gómez-Torres, MJ and Mengual, R.

Department of Biotechnology, University of Alicante

\begin{abstract}
The present study was conducted by using light and transmission electron microscopy to examine the morphologic development of turtle retina from embryonic stage 18 (S18) to S26 (hatching). Particular attention was paid to the formation of functional structures such as neurites, synapses, photoreceptors, among others, and the moment that chemical synapses appear in the outer and inner plexiform layers. The results show that retinal differentiation in the turtle follows the vitreal to scleral morphological differentiation of retinal cells. Moreover, the central region of the optic cup is most advanced compared to the peripheral parts. Early functional plexiform layers, based on appearance of synapses, precede the complete differentiation of photoreceptors. The first synaptic structures occur in the inner plexiform layer before the outer plexiform layer. Receptor outer segments and first synaptic ribbon in receptor synaptic terminals initiate the differentiation at the same time, but final maturation includes dendritic invaginations of bipolar and horizontal cells in the receptor terminals. We assume that at birth, the turtle retina has achieved the ability to see.
\end{abstract}

Keywords: Trachemys scripta elegans, Embryogenesis, Synaptogenesis, Retinal development, ultrastructure 


\section{INTRODUCTION}

As in all vertebrates, turtle retina is a laminated structure consisting of three nuclear layers: the outer nuclear layer (ONL) contains cell bodies of the photoreceptors, the inner nuclear layer (INL) contains cell bodies of the bipolar, horizontal and amacrine neurons and Müller glia, and the ganglion cell layer (GCL) contains cell bodies of ganglion cells and displaced amacrine cells. These layers are separated by two layers of synapses: outer and inner plexiform layers. The thin outer plexiform layer (OPL) contains connections between photoreceptors and bipolar and horizontal cells, whereas the more complex inner plexiform layer (IPL) contains the synapses between the bipolar, amacrine and ganglion cells.

Although the general development of the retina is notably similar in the majority of vertebrates, the temporal course of neurogenesis and synaptic formation processes varies between species. In effect, whereas in some species the retina is entirely developed at birth, in mammals the complete differentiation occurs during postnatal development (Finlay and Sengelaub 1989; Lam and Shatz 1991). This is the difference between altricial and precocial life styles. There are some altricial fish species that also complete retinal differentiation after hatching. Retinal development begins with the initial appearance of distinct cellular types and ends with the establishment of a complex process of synaptic contacts. The onset of synaptogenesis is a key event in the formation of neuronal circuits and structural patterning during neural system development. In contrast to the amazing complexity of the brain, the laminar structure of the retina and its easy accessibility provides a useful model for studies of ultrastructural analysis of synaptogenesis and other aspects of development (Fini et al. 1997; Oliver and Gruss 1997; Schmith and Dowling 1999).

Whereas electrophysiological activity and the ontogenetic expression of neurochemicals in specific populations in the turtle retina during the embryonic development have been studied (Sernargor and Grzywacz 1995; Francisco-Morcillo et al. 2006), little is known about both cell type differentiation and synaptogenesis in the plexiform layers. This work provides a description of the turtle retina maturation process by using light (LM) and transmission electron microscopy (TEM). Particular attention is paid to the development of photoreceptors and the moment that synapses appear in photoreceptor terminals. According Nguyen et al. (2000), our study begins at S18 when the IPL first appears throughout the retina, and it ends with hatching, when function of turtle retina might be achieved. As reptiles are in an intermediate position in phylogeny of vertebrates, this study should contribute to the comparisons of developmental process between other vertebrates.

\section{MATERIALS AND METHODS}

This study was carried out using ten embryos of Trachemys scripta elegans. The following stages were studied: stage (S18), S20, S22, S23, and S26 (hatching), that correspond to 25, 30, 35, 40 and 60 days of incubation, respectively. Turtle eggs were kept in a humid incubator maintained at $29^{\circ} \mathrm{C}$ until their appropriate developmental stage. At this temperature the average incubation time was 60 days. The embryonic ages were determined according to specific stages of turtle development (Yntema 1968). All embryos and hatched animals were treated according to the European Union and the Spanish government regulations and standards.

For ultrastructural study, the retinas were fixed overnight at $4^{\circ} \mathrm{C}$ with $1 \%$ paraformaldehyde, $1.6 \%$ glutaraldehyde, and $0.15 \mathrm{mM} \mathrm{CaCl} 2$ in $0.1 \mathrm{M}$ phosphate buffer $(\mathrm{pH}$ 7.4). After fixation, retinas were rinsed in $5 \%$ sucrose phosphate buffer and postfixed in $2 \%$ OsO4 for $1 \mathrm{hr}$. The retinas were subsequently dehydrated in ethanol, embedded in Epon-812 and sectioned vertically by a (Leica) LKB-III ultramicrotome. Sections of 50 - 
$70 \mathrm{~nm}$ thickness were stained with uranium acetate and lead citrate and viewed with a JEOL model JEM-1400 Plus equipped with a digital camera GATAN ORIUS for acquisition of images. Furthermore, semithin sections $(1 \mu \mathrm{m})$ for LM were stained using toluidine blue. Photomicrographs were taken with a Leica DMRB equipped with a Lumenera's INFINITY microscopy camera.

\section{RESULTS}

The differentiation of Trachemys scripta elegans retina starts in a region close to the centre of optic cup, so that in each stage we can observe various phases of differentiation in each section. As a result for each stage we describe which structures are most advanced in cellular development.

\section{Stage 18}

At S18 retinal lamination is not totally developed (Figure 1A). The retinal pigment epithelial cells (RPE), a cuboidal layer filled with pigment granules, are clearly distinguishable (Figure 1B). The presumptive GCL, INL and ONL are observed. At this stage, the OPL is an irregular stripe that is not evident in all regions, but TEM allows for observing neural processes interspersed with intercellular spaces in an incipient OPL (Figure 1C). The photoreceptors are undeveloped: the inner segment is a cytoplasmic bud in the distal retina that appears to be pushed through the future outer limiting membrane (OLM) (Figure 1B). In effect, the receptor cells present desmosome-like cell junctions in the region of the inner segments. They are characterised by the presence of a lipid droplet, abundant mitochondria, numerous free ribosomes, endoplasmic reticulum and a ciliary structure at the lateral part of photoreceptors. In regions where OPL is observed, three or four rows of nuclei constitute the ONL (Figure 1A).

The INL constitutes the main part of the retina and includes a large population of two clearly distinguishable nuclei (Figure 1A, D). Close to the OPL there are dark nuclei with a concentrated chromatin: the future bipolar and the horizontal cells. Light nuclei of amacrine cells are located close to the IPL, which is a lax reticulum of interlaced immature neural processes with large spaces known as glial channels Moreover, the IPL shows membranes of neighboring processes in close apposition following a parallel trajectory as the prelude to possible future synaptic junctions (Figure 1E). Some contacts present an electron-dense material in the cell membrane but true synaptic structures could not be observed. Also scattered in the early IPL there are abundant mounds of agramular vesicles which may be related to the growing ganglionar axons cells.

Finally, the GCL comprises four or five nuclei rows (Figure 1A) separated by intercellular spaces. The lighter nuclei are similar to the amacrine cells nuclei. In the retinal nerve fibre layer (NFL), axons are abundant but they do not form bundles (Figure 1F).

\section{Stage 20}

The RPE has increased in size so that the area covered by the pigment epithelial cells is bigger than S18 (Figure 2A). The cell membrane at the base of these cells facing Brunch's membrane shows marked infoldings (Figure $2 \mathrm{~B}$ ), which are usually found in cells involved in absorption of substances form adjacent blood vessels. The apical membrane, facing the photoreceptors, shows large microvilli around photoreceptors. These microvilli are well observed in large spaces that occur between pigment epithelial cells and developing inner segments of photoreceptors. Many melanin granules are found in the epithelial cells, which are round and elliptical in shape, 
and cytoplasmic organelles are present, including free ribosomes, mitochondria and rough and smooth endoplasmic reticulum.

The most conspicuous change at S20 is the appearance of a well-defined OPL (Figure 2A), and the elongation and differentiation of a photoreceptor inner segment (Figure 2C). Although the outer segment is absent, the inner segments of many photoreceptors show the characteristic oil droplet, an incipient paraboloid, mitochondria and rough endoplasmic reticulum. Ciliary structure is evident (Figure 2D) in inner segments extending toward the pigment epithelium prior to formation of the outer segment. Lightly and finely extended processes of Müller cells and apical junctions with photoreceptor cells are very evident, so that, a well-differentiated OLM can be observed (Figure 2F). Photoreceptor terminals are immature, and no ribbon synapses are present, but narrowcleft basal junctions and membrane densities are frequently observed (Figure 2E). The OPL mostly shows profiles of neurites of presumably horizontal cell dendrites.

The INL showed several rows of nuclei corresponding to somata of horizontal, bipolar amacrine and Müller cells. Round and lighter nuclei of horizontal cells are located near the OPL whereas nuclei of bipolar cells are darker and located in several layers (Figure 2A). Rough endoplasmic reticulum is continuous with a nuclear envelope of horizontal cells (Figure 2G), and the abundant ribosomes are arrayed in a spiral order. Müller cells are recognisable by their irregular and dark nuclei, and the lighter cytoplasm around them contains abundant glycogen (Figure $2 \mathrm{H}$ ). These cells extend the cytoplasm towards the outer layers. Intercellular spaces have decreased with regard to S18, and the debris of degenerating cells is especially abundant. In the most proximal INL, four rows of nuclei of presumably amacrine cells are observed. These cells are distinguished by clear staining cytoplasm.

The IPL attains considerable thickness through the formation of numerous neural processes assembling dendrites, without intercellular spaces (Figure 2I). There is a presence of abundant presumptive axon terminals of bipolar and amacrine cells containing synaptic vesicles. Profiles of mounds of agranular vesicles are less abundant that in S18, but they are still observed scattered throughout the IPL. In contrast, apoptosis is frequent, considering the abundance of structures with a multilamelar aspect that correspond to degenerative processes. The most striking feature in IPL is the appearance of some structures resembling conventional synapses and occasional synaptic ribbons (Figure 2J). Moreover, some neural processes show tubules and neurofilaments, although most appear empty. Some dark nuclei in IPL are presumed to be displaced amacrines.

GCL includes two or three rows of large sized ganglion cells, lighter nuclei and a prominent nucleolus (Figure $2 \mathrm{~K})$. Extracellular space between ganglion cells exists but is less apparent than S18. In NFL we can distinguish bundles of ganglion cells axons which are separated by end feet of Müller cells (Figure 2L).

Stage 22

The RPE does not show any differences with regard to the previous stage of development (Figure $3 \mathrm{~A}$ ). On the contrary, photoreceptors increase in size and the differentiation is more advanced. Effectively, the photoreceptor outer segment starts developing and the disks, separated by wide spaces, are oriented at many angles relative to the photoreceptor's long axis. Often the ciliary structure from the lateral part of the inner segment is accompanying the outer segment. The inner segments enlarge enormously and show an oil droplet, a paraboloid and an ellipsoid with abundant mitochondria. Inner segments are separated and surrounded by microvilli of the Müller cells, which project beyond the outer limiting membrane. The nuclei of photoreceptors are bounded by 
cisternae of rough endoplasmic reticulum and abundant ribosomes, and glycogen granules accumulate between the endoplasmic reticulum (Figure $3 \mathrm{~B}$ ).

Doubles cones have been appearing (Figure 3C). Both members are intimately opposed along inner segments, and typically, the nucleus of the single member protrudes beyond the outer limiting membrane. Pale Müller cell profiles are located around double cones and typically, mitochondria have accumulated close to the OML (Figure 3D).

The OPL reduces the intercellular space and junctional specializations increase in different parts of the terminal photoreceptors. Even with LM, it is possible to observe the axons of photoreceptors and a row formed by cone pedicles (Figure 3A). TEM permits three different zones to be distinguished in the OPL: an external zone containing lightly branching processes of the Müller cells between the axons of photoreceptors (Figure $3 \mathrm{E}$ ) and below the external zone a dark middle zone with synaptic terminals of photoreceptors can be perceived and, finally, an internal zone composed of bipolar, horizontal and Müller cell processes where coated protrusions, that seem early bouton synapses, are approaching the differentiating membranes in the postsynaptic process (Figure 3F).

Between nuclei of photoreceptors and processes of Müller cells, pedicles appear, showing the characteristic pyramidal shape and a darkly stained cytoplasm. These terminals are filled with glycogen granules, free ribosomes, microtubules and synaptic vesicles. But the most striking characteristic is the presence of many immature synaptic ribbons that don't appear associated with post synaptic processes; ribbon triads are not present at this stage. These ribbons lack synaptic vesicles around them and they are oriented in different ways (Figure 3G).

In the INL the apoptotic process, although it is present, is less abundant than those of S20. In the IPL, the presence of membrane densities, synaptic ribbons and degenerating processes are increased. GCL includes four or five rows of ganglion cells with wide intercellular spaces between them. Apoptotic cells are observed.

\section{Stage 23}

The RPE reaches far between the outer segments of the photoreceptors, which mature progressively from S23 until birth. The photoreceptor outer segments become very elongated and the outer segment membranes multiply (Figure 4A). Mitochondria in the inner segments increase in number and oil droplets increase in size from S23 to the end of embryonic development. Finally, synaptic vesicles and synaptic ribbons in cone pedicles are more numerous and longer, and some profiles of characteristic triads are observed. Many ribbons are associated with postsynaptic processes and are surrounded by synaptic vesicles (Figure 4B). The terminals touch other terminals laterally without glial processes between them (Figure 4C).

The IPL is widening and it is characterised by the presence of many membrane densities, synaptic ribbons and conventional synapses with abundant vesicular contacts throughout their length and typical synaptic densities (Figure 4D, E).

Ganglion cells are voluminous, with a round nucleus which possesses a disperse chromatin and prominent nucleolus. Their cytoplasm shows organelles typical of a neuron: ribosome mitochondria, and Nissl substance. Their packed axons run internally and parallel to the inner surface of the retina and they form the NFL. 


\section{Stage 26}

At this stage the retina is in its final period of cell differentiation. In a radial section, the retina shows the main characteristics of an adult retina. LM observation does not reveal great changes with regard to S23, except for the further widening of the IPL and the elongation of the photoreceptor outer segments (Figure 5A), in which under TEM, membrane discs display a regular appearance (Figure 5B). The photoreceptors are primarily single and double cones with oil droplets. Unequal double cones are formed by a long principal and short accessory cones in apposition and parallel to each other. The inner segment of the short accessory cone lacks a droplet and has a distinctive paraboloid, a glycogen storage body placed between the mitochondrial pack. Rods have larger outer segments than cones and lack oil droplets (Figure 5C).

Photoreceptor synaptic terminals increase and the triads are abundant. Synaptic ribbons, accompanied by synaptic vesicles, are more frequent than in S23 (Figure 5D). The IPL is wider and more developed than S23 (Figure 5E). The GCL contains two layers of cell bodies and Müller cell end feet divide bundles of ganglion cell axons (Figure 5F).

\section{DISCUSSION}

Embryogenesis in the turtle Trachemys scripta elegans lasts about 60 days (Yntema 1968). In this study we describe the retinal maturation in this turtle from the embryonic stage 18 to the birth. Previous studies indicate that at about S14 the retina has initiated its development but until S15 it has neither plexiform nor nuclear layers (Nguyen et al. 2000). Nevertheless, some major classes of cells, such as the cholinergic and GABAergic amacrine cells, have begun to produce neurotransmitters and are migrating into position (Nguyen et al. 2000; Nguyen and Grzywacz 2000).

The process of differentiation of retinal cells in Trachemys scripta elegans retina starts in the internal retina and proceeds to the external retina. These results confirm the observations in other vertebrates (Grun 1982), such as chickens (Morris et al. 1976), pigeons (Bagnoli et al. 1985), shrews (Foelix et al. 1987), monkeys (Hendrickson 1996) and zebrafish (Schmitt and Dowling 1999). At embryonic stage 18, it is poorly differentiated and lamination is incomplete, because the OPL is still incipient. The outer retina consists of the RPE and the developing photoreceptor inner segment. Development continues in the scleral direction with the formation of an evident layer of neuropil corresponding to the OPL at S20. Moreover, the central region of the optic cup is most advanced compared to the peripheral parts. This observation has been reported in other vertebrates (Schmitt and Dowling, 1999; Nguyen and Grzywacz, 2000; Francisco-Morcillo, 2006).

\section{Differentiation of retinal cells and lamination}

Differentiation of retinal cells occurs during the whole of development. Ganglion cells are the first to differentiate and produce neurites and apical dendrites, so that a neuropil-rich layer, the IPL, appears. This layer is a product of the arborisation ganglion cell dendrites and the axons of the amacrine cells, which present signs of differentiation: mitochondria, free ribosomes, and endoplasmic reticulum. Later, at S20, the maturation of the inner nuclear layer is reflected in the complexity of the inner plexiform layer, probably due to the differentiation of bipolar cells and their processes. On the other hand, the development of the outer plexiform layer is evidenced by the growth of dendrites of future bipolar and horizontal cells. 
Although the photoreceptors start rather early, they are the last cells to complete the morphological differentiation. In effect, at S18 apical cytoplasm of the future photoreceptor inner segment protrudes in order to make contact with the RPE and show signs of differentiation. But the outer segments and receptor terminal synapses are the last structures to mature; moreover, they develop within a restricted period of time: between S22 and S23. Maturation of outer segments and formation of terminal receptors and the receptor-bipolarhorizontal cell synapses indicates the full differentiation of the photoreceptor. This also occurs in Xenopus (Kinney and Fisher 1978), chickens (Meller 1984), mice (Olney 1968), cats (Morrison 1983), and dogs (Miller et al. 1989). Subsequently, from S23 to birth, major changes that occur in the photoreceptors are increasing its size, the growing organisation of the outer segment and the maturation and growth of lipid droplets. This growth of the photoreceptor triggers the emergence of light responses in several species, including turtle (Sernagor and Grzywacz 1995) and humans (Breton et al. 1995; Nusinowitz et al. 1998). We supposed that the ability to function is achieved in S23 when both outer segment and synaptic terminals have achieved their final aspect. It is at this time that photoreceptors have the structure to connect to the rest of the retina. Although it is difficult to determine when the turtle retina is able to receive and transmit impulses, at S23, the turtle retina has the morphological substrate for assuming the physiological function.

\section{Synaptogenesis}

In Trachemys scripta elegans the IPL appears before the OPL and synaptogenesis of the IPL seem to mature before that of the OPL. This result has also been reported for peripheral retina of pigeons (Bagnoli et al. 1985), chickens (Morris et al. 1976; Drenhaus et al. 2007), Maccaca mulata (Smelser et al. 1974), tree shrews (Foelix et al. 1987), and monkeys (Hendrickson 1996). However, this finding contrasts with those of other animals: in rodents synapses formed in the OPL slightly earlier than in the IPL. Photoreceptor ribbon synapses appear before bipolar-cell ribbon synapses in zebrafish (Schmitt and Dowling 1999) and cats (Maslim and Stone 1986), and before amacrine-cell conventional synapses in monkey fovea (Hendrickson 1996). As in turtle retina, conventional synapses form in the IPL before the ribbon synapses characteristics of bipolar cells in mice (Blanks et al. 1974; Fisher 1979), rabbits (McArdle et al. 1977), guinea pigs (Spira 1975) and ferrets (Greiner and Weidman 1980).

At S18 the neuropil of the IPL is basically formed by a network of large extracellular spaces, filled with interstitial fluid and flanked by extensions from immature cells that are located on both sides of the IPL. The ends of these extensions follow a parallel path and establish close contact, indicating possible future synapses. These contacts, lacking synaptic vesicles, called by Jacobson (1991) contacting processes, may correspond to filopodial contacts arising from both glial cells (Cornell-Bell et al. 1990; Asou et al. 1994) and growth cones of neuroblasts. This aspect of the neuropil with large lacunae is usually observed with TEM in the early stages of embryonic development of the retina and optic nerve (Kuwabara and Weidman 1974; Greiner and Weidman 1980, 1981; Horsburgh and Sefton 1986; Fitzgibbon and Reese 1992). Indeed, Horsburgh and Sefton (1986), observed large extracellular spaces, called glial channels, which were interpreted as a prelude to the formation, growth and future occupation of axons. Something similar had already been observed in the mouse retina (Silver and Robb 1979; Silver and Sidman 1980; Silver and Sapiro 1981) and in chickens (Krayanek and Goldberg 1981). 
It has been established that formation of conventional synapses in the IPL is accompanied by cellular degeneration and the presence of large extracellular spaces (Greiner and Weidman 1980; Kuwabara and Weidman 1974; Silver and Sidman 1980; Segovia 2015). In effect, synaptogenesis is accompanied by two concomitant processes: cell degeneration and the formation of large extracellular spaces immediately prior to synapse formation. The simplest interpretation of these features is that cells or their processes at the developing plexiform layer are eliminated in order to create extracellular space into which processes of differentiating cells can grow. Additionally, the mounds of agranular vesicles noted above associated with early stages of synapse formation were noted in previous studies of the developing retina (Greiner and Weidman 1980) and were interpreted as grown cones.

In Trachemys scripta elegans, appearance of all types of synapses precedes maturation of photoreceptor outer segments, as has been reported in cats (Maslim and Stone 1986), tree shrews (Foelix et al. 1987), and humans (van Driel et al. 1990). However, although membrane thickening or synaptic ribbons are an indication of a synaptic site, we do not observe synaptic vesicles at the beginning. In contrast, the formation of the first photoreceptor outer segments coincides with the development of the first conventional synapses within the IPL in zebrafish (Schmitt and Dowling 1999).

This study shows that gap junctions between pedicles are formed prior to chemical synapse formation. In OPL, at S20 the formation of narrow cleft basal junctions occurs, when ribbons are scant. This has been reported previously in other vertebrates (Cook and Becker 1995) such as chick (Drenhaus et al. 2007) and cat retinas (Maslim and Stone 1986). Moreover, the maturation of chemical synapses in OPL proceeds when full maturation of the photoreceptors occurs, and the outer segment is formed. This feature has been reported in both rodent and human retinas (Smelser et. al 1974). At S23 many ribbons and synaptic vesicles are associated with postsynaptic processes of horizontal and bipolar cells. These findings support that light responses emerge at S23 in electrophysiological experiments (Sernargor and Grzywacz, 1995). Our results suggest that the retina of Trachemys scripta elegans has achieved the ability to see at birth.

\section{CONCLUSIONS}

The neurogenesis process of turtle retina involves a series of spatial and temporal coordinated events, such as cell proliferation in different regions, migration, differentiation, formation of specific connections and cell death. This process is comparable to all vertebrates: The differentiation of the receptors begins with the formation of the inner segment.

As Barnstable (1987) has pointed out, apparently, lamination and cell differentiations are independent events so that amacrine and ganglion cells initiate differentiation before the formation of different layers. To summarise, although various developmental processes occur at the same time in different retinal layers, the sequence of cell differentiation in turtle retina from S18 to hatching is as follows:

1) Ganglion cell - inner plexiform layer- amacrine cells -photoreceptor inner segments

2) Outer plexiform layer-horizontal cells-bipolar cells-Müller cells

3) Photoreceptor outer segments-photoreceptor terminals

4) Photoreceptor synapses-maturation of outer plexiform layer 
Further investigation would be interesting in order to discover the sequence of development of non-neural parts of the eye such as the lens, and other parts of SNC such as optic tectum in order to infer when a turtle can see. Perhaps turtle retina is able to see at S23 from a neurophysiologic point of view, but other structures are required.

\section{REFERENCES}

Asou H, Hamada K, Uyemura K, Sakota T, Hayashi K (1994) How do oligodendrocytes ensheath and myelinate nerve fibers? Brain Res Bull 35(4):359-65

Bagnoli P, Porciatti V, Lanfranchi A, Bedini, C (1985) Developing pigeon retina: light-evoked responses and ultrastructure of outer segments and synapses. J Comp Neurol 235:384-394

Barnstable CJ (1987) A molecular view of vertebrate retinal development. Mol Neurobiol 1:9-46

Blanks JC, Adinolfi, AM, Lolley RN (1974) Synaptogenesis in the photorrecpetor terminal of the mouse retina. J Comp Neurol 156: 81-94

Breton ME, Quinn, GE, Schueller AW (1995) Development of electroretinogram and rod phototransduction response in human infants. Investi Ophth Vis Sci 36:1588-1602

Cook JE, Becker DL (1995) Gap Junctions in the vertebrate retina. Microsc Res Techniq 31:408-419

Cornell-Bell AH, Thomas PG, Smith SJ (1990) The excitatory neurotransmitter glutamate causes filopodia formation in cultured hippocampal astrocytes. Glia 3(5):322-34

Drenhaus U, Voigt T, Rager G (2007) Onset of synaptogenesis in the plexiform layers of the chick retina: a transmission electron microscopic study. Microsc Res Techniq 70:329-335

Fini ME, Strissel KJ, West-Mays JA (1997) Perspectives on eye development. Dev Genet 20:175-185

Finlay BL, Sengelaub DR (1989) Development of the vertebrate retina. Plenum Press, New York

Fisher LJ (1979) Development of synaptic arrays in the inner plexiform layer of neonatal mouse retina. J Comp Neurol 187:359-372

Fitzgibbon T, Reese BE (1992) Position of growth cones within the retinal nerve fibre layer of fetal ferrets. J Comp Neurol 323(2):153-66

Foelix RF, Kretz R, Rager G (1987) Structure and postnatal development of photoreceptors and their synapses in the retina of the tree shrew (Tupaia belangeri). Cell Tissue Res 247:287-297

Francisco-Morcillo J, Hidalgo-Sánchez M, Martín-Partido G (2006) Spatial and temporal patterns of proliferation and differentiation in the developing turtle eye. Brain Res 1103(1):32-48

Greiner JV, Weidman TA (1980) Histogenesis of the cat retina. Exp Eye Res 30:439-453

Greiner JV, Weidman TA (1981) Histogenesis of the ferret retina Exp Eye Res 33:315-32

Grun GG (1982) The development of the vertebrate retina: A comparative survey. Adv Anat Embryol Cel 78:184 
Hendrickson AE (1996) Synaptic development in macaque monkey retina and its implications for other developmental sequences. Perspect Dev Neurobi 3:195-201

Horsburgh GM, Sefton AJ (1986) The early development of the optic nerve and chiasm in embryonic rat. J Comp Neurol 243(4):547-60

Jacobson M (1991) Developmental Neurobiology. New York: Plenum Press

Kinney MS, Fisher SK (1978) The photoreceptors and pigment epithelium of the larval Xenopus retina: morphogenesis and outer segment renewal. P Roy Soc B-Biol Sci 201:149-167

Krayanek S, Goldberg S (1981) Axonal guidance during development of the optic nerve: the role of pigmented epithelia and other extrinsic factors. J Comp Neurol 202(4):521-38

Kuwabara T, Weidman TA (1974) Development of the prenatal rat retina. Invest Ophth Visual 13:725-739

Lam DMK, Shatz CJ (1991) Development of the visual system. MIT Press, Cambridge Massachusetts

Maslim J, Stone J (1986) Synaptogenesis in the retina of the cat. Brain Res 373:35-48

McArdle CB, Dowling JE, Masland RH (1977) Development of outer segments and synapses in the rabbit retina. J Comp Neurol 175:253-274

Meller K (1984) The ultrastructure of the developing inner and outer segments of the photoreceptors of chick embryo retina as revealed by the rapid-freezing and deep-etching techniques. Anat Embryol 169:141-150

Miller WW, Albert RA, Boosinger TR, Holloway CL, Simpson ST, Tojvio-Kinnucan MA (1989) Postnatal development of the photoreceptor inner segment of the retina in dogs. Am J Vet Res 50:2089-2092

Morris VB, Wylie CC, Miles VJ (1976) The growth of the chick retina after hatching. Anat Rec 184:111-113

Morrison JD (1983) Morphogenesis of photoreceptor outer segments in the developing kitten retina. J Anat 136: 521-33

Nguyen LT, Grzywacz NM (2000) Co-Localization of Choline Acetyltransferase and GABA in the Developing and Adult Turtle Retinas. J Comp Neurol 420:527-538

Nguyen LT, De Juan J, Mejia M, Grzywacz, NM (2000) Localization of Choline Acetyltransferase in the Developing and Adult Turtle Retinas. J Comp Neurol 420:512-526

Nusinowitz S, Birch DG, Birch EE (1998) Rod photoresponses in 6-week and 4-month-old human infants. Vision Res 38:627-635

Oliver G, Gruss P (1997) Current views on eye development. Trends Neurosci 20:415-421

Olney JW (1968) An electron microscopic study of synapse formation, receptor outer segment development, and other aspects of developing mouse retina. Invest Ophth Vis Sci 7:250-268

Schmitt EA, Dowling JE (1999) Early retinal development in the zebrafish, Danio rerio: light and electron microscopic analyses. J Comp Neurol 404:515-356

Segovia Y (2015) Apoptosis in turtle embryonic retina (Trachemys scripta elegans). Int J Morphol 33(1):119129

Sernagor E, Grzywacz NM (1995) Emergence of complex receptive field properties of ganglion cells in the developing turtle retina. J Neurophysiol 73:1355-1364 
Silver J, Robb RM (1979) Studies on the development of the eye cup and optic nerve in normal mice and in mutants with congenital optic nerve aplasia. Dev Biol 68(1):175-90

Silver J, Sapiro J (1981) Axonal guidance during development of the optic nerve: the role of pigmented epithelia and other extrinsic factors. J Comp Neurol 202(4):521-38

Silver J, Sidman RL (1980) A mechanism for the guidance and topographic patterning of retinal ganglion cell axons. J Comp Neurol 189(1):101-11

Smelser GK, Ozanics V, Rayborn M, Sagun D (1974) Retinal synaptogenesis in the primate. Invest Ophth Visual 13(5):340-361

Spira AW (1975) In utero development and maturation of the retina of a non-primate mammal: a light and electron microscopic study of the guinea pig. Anat Embryol 146:279-300

van Driel D, Provis JM, Billson FA (1990) Early differentiation of ganglion, amacrine, bipolar, and Muller cells in the developing fovea of human retina. J Comp Neurol 291:203-219

Yntema CL (1968) A series of stages in the embryonic development of Chelydra serpentina. J Morphol $125: 219-251$

ACKNOWLEDGEMENTS: This research was supported by University of Alicante VIGROB-186 